\title{
AMENDMENTS
}

\section{Author Correction: High systemic and tumor-associated IL-8 correlates with reduced clinical benefit of PD-L1 blockade}

Kobe C. Yuen, Li-Fen Liu, Vinita Gupta, Shravan Madireddi, Shilpa Keerthivasan, Congfen Li, Deepali Rishipathak, Patrick Williams, Edward E. Kadel IIID, Hartmut Koeppen, Ying-Jiun Chen, Zora Modrusan, Jane L. Grogan (D), Romain Banchereau (D), Ning Leng, AnnChristine Thastrom, Xiadong Shen, Kenji Hashimoto, Darren Tayama, Michiel S. van der Heijden, Jonathan E. Rosenberg, David F. McDermott (D), Thomas Powles, Priti S. Hegde, Mahrukh A. Huseni (D) and Sanjeev Mariathasan (D)

Correction to: Nature Medicine https://doi.org/10.1038/s41591-020-0860-1, published online 11 May 2020.

In the version of this article initially published, three accession numbers (EGAS00001004229, EGAS00001004386 and EGAS00001004387) were missing from the Data Availability Statement in the HTML. The correct statement is as follows: "Raw data analyzed in this study have been submitted to the European Genome-Phenome Archive with accession numbers EGAS00001004008, EGAS00001004229, EGAS00001004230, EGAS00001004386 and EGAS00001004387." The error has been corrected in the HTML version of the article. Also, the original Reporting Summary file has been replaced with a revised file that includes these accession numbers.

Published online: 5 February 2021

https://doi.org/10.1038/s41591-021-01246-4

๑) The Author(s), under exclusive licence to Springer Nature America, Inc. 2021

\section{Author Correction: Sampling the host response to SARS-CoV-2 in hospitals under siege}

Alexander W. Charney, Nicole W. Simons, Konstantinos Mouskas, Lauren Lepow, Esther Cheng, Jessica Le Berichel, Christie Chang (D), Robert Marvin, Diane Marie Del Valle, Sharlene Calorossi, Alona Lansky, Laura Walker, Manishkumar Patel, Hui Xie, Nancy Yi, Alex Yu, Gurpawan Kang (D), Anthony Mendoza, Lora E. Liharska, Emily Moya, Matthew Hartnett, Sandra Hatem, Lillian Wilkins, Melody Eaton, Hajra Jamal, Kevin Tuballes, Steven T. Chen, Alexandra Tabachnikova, Jonathan Chung, Jocelyn Harris, Craig Batchelor, Jose Lacunza, Mahlet Yishak, Kimberly Argueta, Neha Karekar, Brian Lee, Geoffrey Kelly, Daniel Geanon, Diana Handler, John Leech, Hiyab Stefanos, Travis Dawson, leisha Scott, Nancy Francoeur, Jessica S. Johnson, Akhil Vaid, Benjamin S. Glicksberg (D), Girish N. Nadkarni, Eric E. Schadt, Bruce D. Gelb, Adeeb Rahman, Robert Sebra, Glenn Martin, The Mount Sinai COVID-19 Biobank Team*, Thomas Marron, Noam Beckmann, Seunghee Kim-Schulze, Sacha Gnjatic (D) and Miriam Merad

Correction to: Nature Medicine https://doi.org/10.1038/s41591-020-1004-3, published online 27 July 2020.

In the version of this article initially published, an author ('Sasha Tabachnikova') was incorrectly listed in the Mount Sinai COVID-19 Biobank Team list but should be in the initial author list (as 'Alexandra Tabachnikova'), and another author (Anthony Mendoza) was omitted from the initial author list. The corrected initial author list for the inclusion of these authors is as follows: “...Gurpawan Kang ${ }^{1}$, Anthony Mendoza ${ }^{1}$, Lora E. Liharska ${ }^{1}, \ldots$ Steven T. Chen ${ }^{1}$, Alexandra Tabachnikova ${ }^{1}$, Jonathan Chung ${ }^{1}, \ldots .$. A third author (Alara Akyatan) was omitted from the Mount Sinai COVID-19 Biobank Team list. That corrected author list for the inclusion of this author is as follows: “...Priyal Agrawal ${ }^{1}$, Alara Akyatan ${ }^{1}$, Kasey Alesso-Carra ${ }^{1}, \ldots$. . The errors have been corrected in the HTML and PDF versions of the article.

${ }^{\star}$ A list of authors and their affiliations appears online.

Published online: 5 February 2021

https://doi.org/10.1038/s41591-021-01247-3

(c) The Author(s), under exclusive licence to Springer Nature America, Inc. 2021 